\title{
A Box Spline Calculus for the Discretization of Computed Tomography Reconstruction Problems
}

\author{
Alireza Entezari*, Member, IEEE, Masih Nilchian, Student Member, IEEE, and Michael Unser, Fellow, IEEE
}

\begin{abstract}
B-splines are attractive basis functions for the continuous-domain representation of biomedical images and volumes. In this paper, we prove that the extended family of box splines are closed under the Radon transform and derive explicit formulae for their transforms. Our results are general; they cover all known brands of compactly-supported box splines (tensor-product B-splines, separable or not) in any number of dimensions. The proposed box spline approach extends to non-Cartesian lattices used for discretizing the image space. In particular, we prove that the 2-D Radon transform of an $N$-direction box spline is generally a (nonuniform) polynomial spline of degree $N-1$. The proposed framework allows for a proper discretization of a variety of tomographic reconstruction problems in a box spline basis. It is of relevance for imaging modalities such as X-ray computed tomography and cryo-electron microscopy. We provide experimental results that demonstrate the practical advantages of the box spline formulation for improving the quality and efficiency of tomographic reconstruction algorithms.
\end{abstract}

Index Terms-B-splines, box splines, computed tomography, Radon transform.

\section{INTRODUCTION}

B IOMEDICAL imaging heavily relies on tomographic algorithms for the reconstruction of 2-D and 3-D images from projection data [19]. Prominent medical imaging examples are X-ray computed tomography, emission tomography [positron emission tomography (PET) and single-photon emission computed tomography (SPECT)], and portal imaging for radio therapy. Tomographic reconstruction is also relevant for biology, for instance, for small animal imaging using microscanners, optical projection tomography, and molecular structure determination from 3-D cryo-electron tomography.

When the projection angles are evenly distributed, the tomogram is usually reconstructed by filtered back-projection (FBP) [20], [19]. When the acquisition conditions are less ideal (e.g., noisy and/or missing data, noneven angular distribution of the projections), it is better to apply iterative techniques such as al-

Manuscript received December 24, 2011; revised March 08, 2012; accepted March 08, 2012. Date of publication March 20, 2012; date of current version July 27,2012 . This work was supported in part by the U.S. National Science Foundation under Grant CCF/CIF-1018149, in part by the Swiss National Science Foundation under Grant 200020-109415, and in part by the Center for Biomedical Imaging of the Geneva-Lausanne Universities and EPFL. Asterisk indicates corresponding author.

*A. Entezari is with the Computer and Information Science and Engineering Department, University of Florida, Gainesville, FL 32611 USA (e-mail: entezari@cise.ufl.edu).

M. Nilchian and M. Unser are with the Biomedical Imaging Group, Ecole Polytechnique Federale de Lausanne, CH-1015 Lausanne, Switzerland (e-mail: masih.nilchian@epfl.ch; michael.unser@epfl.ch).

Digital Object Identifier 10.1109/TMI.2012.2191417 gebraic reconstruction (ART) or the statistical OSEM method, which offer greater flexibility.

A crucial step in the design of iterative reconstruction algorithms is the discretization of the forward model. This is usually achieved by selecting image-domain basis functions and by mathematically simulating the acquisition process on them. For a parallel projection geometry, the suitable model is the Radon transform (or the X-ray transform in 3-D). Current reconstruction algorithms typically use the natural (square) pixel basis or some blob (or Kaiser-Bessel) functions which have the advantage of being circularly symmetric. While these particular choices simplify the derivation of the forward model, they are not as favorable from an approximation theoretic point of view. Both bases have — at best — a first order of approximation which implies that the rate of decay of the discretization error as the reconstruction grid gets finer is relatively slow [17], [23]. In principle, choosing higher order B-spline basis functions would be more advantageous since these are optimal in the sense of their support being minimal for a given order of approximation [23]. Splines have been found to be useful for improving the performance of FBP reconstruction [14], [12], [22] but have hardly been deployed in the context of iterative algorithms, probably due to the increased complexity of the corresponding forward model.

Recently, splines have been used for determining the Hilbert transform of the sinogram signal, instead of the traditional Fourier-based method [6], [7] which is widely used for FBP.

The problem of reconstructing a multivariate polynomial from its Radon projections is studied in [1]. This problem offers an approximation-theoretic view for the analysis of $\mathrm{X}$-ray tomography algorithms. Moreover, using the orthogonal expansion of multivariate polynomials (e.g., Chebyshev expansions), it is possible to provide partial sum approximations to the tomography problem along with closed-form error analysis [26]. Multivariate polynomials can be expanded in box spline basis (via Marsden's identity), as spline spaces spanned by the compactly supported box splines contain multivariate polynomials of certain degrees (up to their approximation order). Hence, our box spline approach offers a direct method for local reconstruction of multivariate polynomials from their Radon or $\mathrm{X}$-ray projections.

The contribution of this paper is to provide a general characterization of the Radon (or X-ray) transform for the extended family of box splines. ${ }^{1}$ While bringing in this level of generality may look like overkill at first sight, we find that it actually simplifies the analysis because the family happens to be

\footnotetext{
${ }^{1} \mathrm{~A}$ preliminary version of this approach with 2-D B-splines was presented at the ISBI conference. [4]
} 


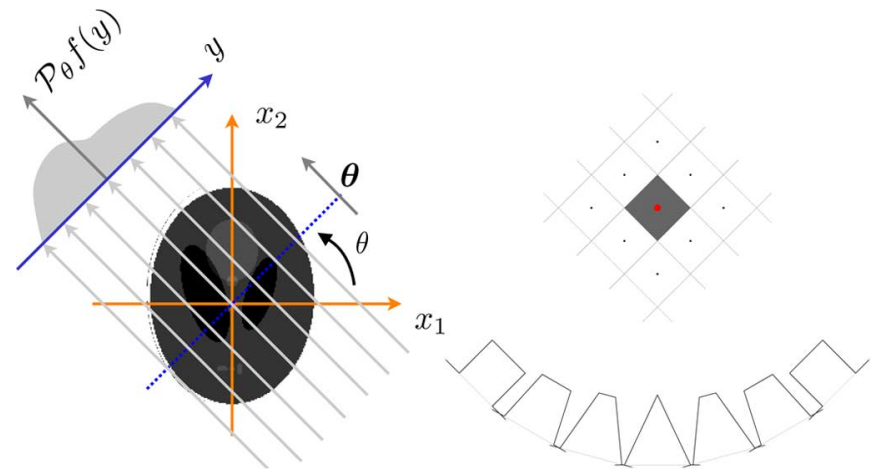

Fig. 1. X-ray projection geometry for $d=2$. On the right: projection of a pixel (tensor-product first-order B-spline) on various angles.

closed under the Radon/X-ray transform. Since all commonly used brands of B-splines are special instances of box splines [2], it therefore makes sense to investigate these functions in detail to obtain a complete analytical picture.

\section{Projection Geometry}

The X-ray transform relates a function to its projections along some directions $\boldsymbol{\theta}$. The projections (or shadows) are obtained by integrating the function along a set of parallel rays. In two dimensions, this is equivalent to performing the Radon transform.

To specify the geometry in the general $d$-dimensional setting, we introduce the unit vector $\boldsymbol{\theta} \in \mathbb{R}^{d}$ that points along the direction of integration. The spatial coordinates of the input function are denoted by $\boldsymbol{x}=\left[x_{1} x_{2} \ldots x_{d}\right]^{T}$. Let $\boldsymbol{P}_{\boldsymbol{\theta}^{\perp}}^{T}$ be a $d \times(d-1)$ matrix specifying an orthogonal basis of the hyperplane perpendicular to the direction of integration $\boldsymbol{\theta}$. The corresponding hyperplane coordinates are $\boldsymbol{y}=\left[y_{1} \ldots y_{d-1}\right]^{T}=\boldsymbol{P}_{\boldsymbol{\theta}^{\perp}} \boldsymbol{x}$; they are obtained by projecting $\boldsymbol{x}$ onto the basis vectors perpendicular to $\boldsymbol{\theta}$. These are also expressed in a rotated coordinate system as

$$
\boldsymbol{x}=t \boldsymbol{\theta}+\boldsymbol{P}_{\boldsymbol{\theta}^{\perp}}^{T} \boldsymbol{y} .
$$

The ( $d$-dimensional) X-ray transform $\mathcal{P}$ maps a function $f(\boldsymbol{x}), \boldsymbol{x} \in \mathbb{R}^{d}$ into the set of its line integrals [19]. Specifically, if $\theta \in S^{d-1}$ (e.g., $\boldsymbol{\theta} \in \mathbb{R}^{d}$ with $\|\boldsymbol{\theta}\|=1$ ), then

$$
\mathcal{P}\{f\}(\boldsymbol{y} ; \boldsymbol{\theta})=\int_{\mathbb{R}} f\left(t \boldsymbol{\theta}+\boldsymbol{P}_{\boldsymbol{\theta}^{\perp}}^{T} \boldsymbol{y}\right) \mathrm{d} t
$$

with $\boldsymbol{y} \in \mathbb{R}^{d-1}$. In the sequel, we will also use the shorthand notation $\mathcal{P}_{\boldsymbol{\theta}} f(\boldsymbol{y})$ for the previous map. The projection geometry for the 2-D case is illustrated in Fig. 1; the direction of integration is $\boldsymbol{\theta}=(-\sin \theta \cos \theta)^{T}$ and the projection matrix onto the Radon coordinate system is $\boldsymbol{P}_{\boldsymbol{\theta}^{\perp}}=\left[\begin{array}{ll}\cos \theta & \sin \theta\end{array}\right]$ so that $y=x_{1} \cos \theta+x_{2} \sin \theta$.

The X-ray transform is a bounded linear operator that has the convenient property of pseudo-commuting with the convolution and translation operations

$$
\begin{aligned}
\mathcal{P}_{\boldsymbol{\theta}}\{f * g\}(\boldsymbol{y}) & =\left(\mathcal{P}_{\boldsymbol{\theta}} f * \mathcal{P}_{\boldsymbol{\theta}} g\right)(\boldsymbol{y}) \\
\mathcal{P}_{\boldsymbol{\theta}}\left\{f\left(\cdot-\boldsymbol{x}_{0}\right)\right\}(\boldsymbol{y}) & =\mathcal{P}_{\boldsymbol{\theta}}\{f\}\left(\boldsymbol{y}-\boldsymbol{P}_{\boldsymbol{\theta}^{\perp}} \boldsymbol{x}_{0}\right) .
\end{aligned}
$$

These properties are essential to the present work where we are aiming at obtaining a closed-form expression for the X-ray transform of any spline or box spline function defined on a regular grid.

\section{DiSCRETIZATION OF RADON TRANSFORM}

To formulate a spline-based forward model for computed tomography, we consider an input function that is expressed in an integer-shift invariant basis

$$
f(\boldsymbol{x})=\sum_{\boldsymbol{k} \in \mathbb{Z}^{d}} c_{\boldsymbol{k}} \varphi(\boldsymbol{x}-\boldsymbol{k})
$$

where the generator $\varphi$ is a tensor-product B-spline or, more generally, a box spline. This is not a restriction because there is usually a one-to-one linear correspondence between the samples of a function (or pixels) and the B-spline coefficients $c_{\boldsymbol{k}}$ [23]. The direct application of (4) then yields

$$
\mathcal{P}_{\boldsymbol{\theta}} f(\boldsymbol{y})=\sum_{\boldsymbol{k} \in \mathbb{Z}^{d}} c_{\boldsymbol{k}} \mathcal{P}_{\boldsymbol{\theta}}\{\varphi\}\left(\boldsymbol{y}-\boldsymbol{P}_{\boldsymbol{\theta}^{\perp}} \boldsymbol{k}\right) .
$$

This means that we can discretize the X-ray reconstruction problem exactly, provided that we have an explicit formula for $\mathcal{P}_{\boldsymbol{\theta}}\{\varphi\}(\boldsymbol{y})$, the X-ray (or Radon) transform of the B-spline generator. It is then possible to use (6) to form the system matrix $\boldsymbol{H}$ that relates the line integrals $p_{m, n}=\mathcal{P}_{\boldsymbol{\theta}_{m}} f\left(\boldsymbol{y}_{n}\right)$ to the B-spline coefficients of the signal $c_{\boldsymbol{k}}$. The corresponding adjoint operator (back-projection process) is represented by $\boldsymbol{H}^{T}$, the transpose of the system matrix.

We also note that the approach is applicable to nonparallel geometries (e.g., fan beam or cone beam), as long as the measurements corresponds to pure line integrals. For example, one can define a geometric transformation where each ray in a fan-beam setup is transformed to a single ray in a parallel geometry. This transformation allows us to compute the ray contribution using $\mathcal{P}_{\boldsymbol{\theta}}\{\varphi\}(\boldsymbol{y})$ in the corresponding parallel geometry.

In practice, one may rely on the analytical forms derived in this paper to precompute the B-spline projections $\mathcal{P}_{\boldsymbol{\theta}} \varphi(\boldsymbol{y})$ with an adequate sampling along the angular and spatial dimensions. An effective way to reduce the algorithmic cost of (6) is by storing an oversampled version of these functions in a table. It is then possible to implement an efficient version of a spline-based forward model (as well as its adjoint = backprojection) using the fact that the influence of a basis function is very local (sparse system matrix). Specifically, one should simply scan through the projection lines hitting the detector, while forming an appropriate linear combination of the B-spline coefficients in (5) with nonzero contributions, that is, the ones for which the support of the B-spline intersects the projection line $(m, n)$. The required weights $[\boldsymbol{H}]_{((m, n), \boldsymbol{k})}$ (nonzero system matrix entries) are retrieved by simple table lookup (nearest neighbor interpolation). The discretization error can be made arbitrarily small by using a sufficient margin of oversampling along the $\boldsymbol{y}$ dimension with no computational penalty.

\section{Box SPlines REVIEW}

Box splines are smooth piecewise polynomial functions defined in $\mathbb{R}^{d}$ that are (nonseparable) generalization of univariate B-splines to the multivariate setting. The definitive reference on the subject is the monograph by de Boor et al. [2], which is rather mathematically oriented. Here, we briefly summarize the 
results of box spline theory that are pertinent to the derivations in this paper. In particular, we emphasize the convolutional interpretation of these functions.

\section{A. Basic Geometric Definition}

Geometrically, a box spline is the shadow (i.e., X-ray image) of a hypercube, in $\mathbb{R}^{N}$, when projected to a lower dimensional space, $\mathbb{R}^{d}(N \geq d)$. A box spline is defined for a set of $N$ vectors $\boldsymbol{\xi}_{1}, \boldsymbol{\xi}_{2}, \ldots, \boldsymbol{\xi}_{N}$ in $\mathbb{R}^{d}$. Each of these vectors is the shadow of an edge of the $N$-hypercube adjacent to its origin. The matrix of directions $\boldsymbol{\Xi}:=\left[\boldsymbol{\xi}_{1} \xi_{2} \ldots \xi_{N}\right]$ completely specifies the box spline in $\mathbb{R}^{d}$. Note that the vectors in this (multi-) set need not be distinct as they can appear with some multiplicity. When $N=d$, the box spline is simply the (normalized) indicator function of the parallelepiped formed by $d$ vectors in $\mathbb{R}^{d}$

$$
M_{\boldsymbol{\Xi}}(\boldsymbol{x})= \begin{cases}\frac{1}{|\operatorname{det} \boldsymbol{\Xi}|}, & \boldsymbol{x}=\sum_{n=1}^{d} t_{n} \boldsymbol{\xi}_{n} \text { for some } 0 \leq t_{n} \leq 1 \\ 0, & \text { otherwise }\end{cases}
$$

For $N>d$, box splines are defined recursively by a "directional" convolution which makes them particularly suitable for the Radon transform

$$
M_{\Xi \cup \boldsymbol{\xi}}(\boldsymbol{x})=\int_{0}^{1} M_{\Xi}(\boldsymbol{x}-t \boldsymbol{\xi}) \mathrm{d} t
$$

When the lower dimensional space is $\mathbb{R}$ (i.e., $d=1$ ), the box splines coincide with univariate B-splines (basic splines). When the distinct column vectors of $\boldsymbol{\Xi}$ are orthogonal to each other, box splines amount to tensor-product B-splines.

The shifts of $M_{\Xi}$ on $\mathbb{Z}^{d}$ form the spline space

$$
S_{M_{\Xi}}:=\operatorname{span}\left(M_{\Xi}(\cdot-\boldsymbol{k})\right)_{\boldsymbol{k} \in \mathbb{Z}^{d}}
$$

If $\kappa$ is the minimal number of directions whose removal from $\boldsymbol{\Xi}$ makes the remaining directions not span $\mathbb{R}^{d}$, then all polynomials up to degree $\kappa-1$ are contained in $S_{M_{\Xi}}$ [2]; also, the approximation order of $S_{M_{\Xi}}$ is $\kappa$. Furthermore, the continuity of the box spline is at least

$$
M_{\boldsymbol{\Xi}} \in C^{\kappa-2}\left(\mathbb{R}^{d}\right)
$$

\section{B. Elementary Box Spline Constituents}

Another way of constructing box splines, which is probably more transparent to engineers, is by repeated convolution of elementary line-segment-like distributions. Specifically, we have

$$
M_{\boldsymbol{\Xi}}(\boldsymbol{x})=\left(M_{\boldsymbol{\xi}_{1}} * \cdots * M_{\boldsymbol{\xi}_{N}}\right)(\boldsymbol{x})
$$

where the elementary box splines, $M_{\boldsymbol{\xi}_{n}}$, are Dirac-like line distributions supported over $\boldsymbol{x}=t \boldsymbol{\xi}_{n}$ with $t \in[0,1]$ with a unit integral. These elementary box splines are in direct geometric correspondence (via a rotation and a proper scaling) with the primary box spline

$$
M_{\vec{e}_{1}}(\boldsymbol{x})=\operatorname{box}\left(x_{1}\right) \delta\left(x_{2}, \ldots, x_{d}\right)
$$

where $\delta\left(x_{2}, \ldots, x_{d}\right)$ is the $(d-1)$-dimensional Dirac distribution and

$$
\operatorname{box}(x)=\left\{\begin{array}{ll}
1, & 0 \leq x \leq 1 \\
0, & \text { otherwise }
\end{array} .\right.
$$

Moreover, they integrate to 1 which is a property that is shared by all box splines (and also preserved through convolution).

Based on (10), one directly infers that the box splines are positive, compactly supported functions. Their support is a zonotope, which is the Minkowski sum of $N$ vectors in $\boldsymbol{\Xi}$. The center of the support of $M_{\Xi}(\boldsymbol{x})$ is given by $\boldsymbol{c}_{\Xi}:=(1) /(2) \sum_{n=1}^{N} \boldsymbol{\xi}_{n}$. The Fourier transform of the box spline is therefore given by

$$
\begin{aligned}
\hat{M}_{\boldsymbol{\Xi}}(\boldsymbol{\omega}) & =\prod_{n=1}^{N} \frac{1-\exp \left(-j\left\langle\boldsymbol{\xi}_{n}, \boldsymbol{\omega}\right\rangle\right)}{j\left\langle\boldsymbol{\xi}_{n}, \boldsymbol{\omega}\right\rangle} \\
& =\exp \left(-j\left\langle\boldsymbol{c}_{\boldsymbol{\Xi}}, \boldsymbol{\omega}\right\rangle\right) \prod_{n=1}^{N} \operatorname{sinc}\left(\frac{\left\langle\boldsymbol{\xi}_{n}, \boldsymbol{\omega}\right\rangle}{2 \pi}\right)
\end{aligned}
$$

where $\omega:=\left[\omega_{1}, \ldots, \omega_{d}\right]^{T}$ is the multivariate frequency vector. Alternatively, a centered box spline, denoted by $M_{\Xi}^{c}$ which is shifted to the origin, has the simple Fourier transform

$$
\hat{M}_{\Xi}^{c}(\boldsymbol{\omega})=\prod_{n=1}^{N} \operatorname{sinc}\left(\frac{\left\langle\boldsymbol{\xi}_{n}, \boldsymbol{\omega}\right\rangle}{2 \pi}\right)
$$

\section{X-Ray Projection of Box Splines}

We now turn to our main objective, which is the derivation of an explicit formula for $\mathcal{P}_{\boldsymbol{\theta}}\left\{M_{\boldsymbol{\Xi}}\right\}(\boldsymbol{y})$ where $M_{\boldsymbol{\Xi}}(\boldsymbol{x})$ is a given box spline generator specified by $N$ direction vectors $\boldsymbol{\xi}_{n} \in \boldsymbol{\Xi}$. In the following discussion, $\boldsymbol{\nu}=\left[\nu_{1}, \ldots, \nu_{d-1}\right]^{T}$ denotes the $(d-1)$-variate frequency vector corresponding to the projection-domain spatial coordinate vector $\boldsymbol{y} \in \mathbb{R}^{d-1}$, while the projection geometry is the specified in Section II.

Theorem 1 (Sinogram-Domain Parameterization): The X-ray transform of a $d$-variate box spline specified by the direction set, $\boldsymbol{\Xi}$, is a $(d-1)$-variate box spline whose direction set, $Z=\left[\zeta_{1} \zeta_{2} \ldots \zeta_{N}\right]$, is the geometric projection of the former. Specifically

$$
\mathcal{P}_{\boldsymbol{\theta}}\left\{M_{\boldsymbol{\Xi}}\right\}(\boldsymbol{y})=M_{\mathbf{P}_{\boldsymbol{\theta}} \perp} \boldsymbol{\Xi}(\boldsymbol{y})
$$

where $\mathbf{P}_{\boldsymbol{\theta}^{\perp}}$ is the transformation matrix that geometrically projects the $\boldsymbol{x}$-coordinate system onto the $\boldsymbol{y}$-coordinate system perpendicular to $\boldsymbol{\theta}$.

Proof: We start with the derivation of the X-ray transform of the elementary (Dirac-type) box spline $M_{\boldsymbol{\xi}}(\boldsymbol{x})$ whose distributional Fourier transform is

$$
\hat{M}_{\boldsymbol{\xi}}(\boldsymbol{\omega})=\frac{1-\exp (\langle\boldsymbol{\xi}, \boldsymbol{\omega}\rangle)}{j\langle\boldsymbol{\xi}, \boldsymbol{\omega}\rangle}
$$

We can proceed geometrically by determining the "shadow" of the direction vector $\xi$ since the latter specifies the support of the elementary box spline as a line segment in $\mathbb{R}^{d}$. The alternative 

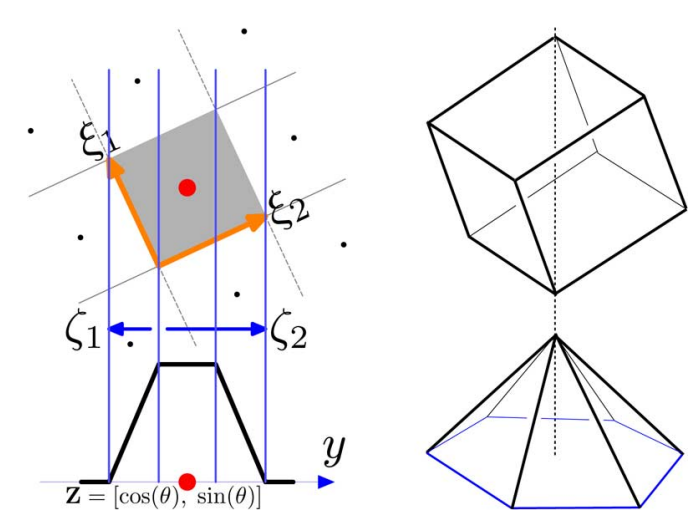

Fig. 2. X-ray transform of a box spline is a box spline whose directions are projections of directions of the original box spline onto the projected plane. On the right: trivariate box spline (tensor-product B-spline) projected to 2-D.

is to apply the central slice theorem which states that the Fourier transform of $\mathcal{P}_{\boldsymbol{\theta}} \varphi(\boldsymbol{y})$ corresponds to the restriction of $\hat{\varphi}(\boldsymbol{\omega})$ to the hyperplane perpendicular to $\boldsymbol{\theta}$. Specifically, we have that

$$
{\widehat{\mathcal{P}_{\boldsymbol{\theta}} M_{\boldsymbol{\xi}}}}_{\boldsymbol{\nu}} \boldsymbol{\nu}=\left.\hat{M}_{\boldsymbol{\xi}}(\boldsymbol{\omega})\right|_{\boldsymbol{\omega}=\mathbf{P}_{\boldsymbol{\theta}}^{T} \boldsymbol{\nu}}=\hat{M}_{\mathbf{P}_{\boldsymbol{\theta}}^{\perp}} \boldsymbol{\xi}(\boldsymbol{\nu}) .
$$

Since $\left\langle\boldsymbol{\xi}, \mathbf{P}_{\boldsymbol{\theta}^{\perp}}^{T} \boldsymbol{\nu}\right\rangle=\left\langle\mathbf{P}_{\boldsymbol{\theta}^{\perp}} \boldsymbol{\xi}, \boldsymbol{\nu}\right\rangle$, we can define the projected directions by $\zeta:=\mathbf{P}_{\boldsymbol{\theta}^{\perp}} \boldsymbol{\xi}$. This allows us to deduce that

$$
\mathcal{P}_{\boldsymbol{\theta}}\left\{M_{\boldsymbol{\xi}}\right\}(\boldsymbol{y})=M_{\mathbf{P}_{\boldsymbol{\theta}} \boldsymbol{\xi}}(\boldsymbol{y})=M_{\boldsymbol{\zeta}}(\boldsymbol{y}) .
$$

This proves the theorem for $N=1$. By defining

$$
\boldsymbol{Z}:=\left[\mathbf{P}_{\boldsymbol{\theta}^{\perp}} \boldsymbol{\xi}_{1} \ldots \mathbf{P}_{\boldsymbol{\theta}^{\perp}} \boldsymbol{\xi}_{N}\right]=\mathbf{P}_{\boldsymbol{\theta}^{\perp}} \boldsymbol{\Xi}
$$

we are then able to transfer the result to the general case using convolution properties (3) and (10).

The theorem is illustrated in Fig. 2. The box spline on the right is a trivariate tensor-product $\mathrm{B}$-spline (first order) whose direction vectors are $(1,0,0),(0,1,0)$ and $(0,0,1)$. When projected to the plane orthogonal to $\boldsymbol{\theta}$, it yields a bivariate, three-direction, box spline that is a hat function with hexagonal support. Likewise, the X-ray transform of the trilinear B-spline (second order) is again a three-direction box spline, but with multiplicity of 2. The concept carries over to higher order tensor-product $\mathrm{B}$-splines which are transformed into three-direction box splines with repeated directions, the main point being that these can be evaluated efficiently.

Corollary 2 (Image-Domain Parameterization): The X-ray transform of an $s$-variate box spline specified by the direction set $\boldsymbol{\Xi}$ along a direction $\boldsymbol{\theta}$ is an $(s-1)$-variate box spline. The directions of the latter $(s-1)$-variate box spline are obtained by geometric projection of the directions $\xi \in \boldsymbol{\Xi}$ into the (hyper) plane orthogonal to the projection direction $\boldsymbol{\theta}$

$$
\zeta=\xi-\langle\xi, \theta\rangle \theta .
$$

Corollary 3 (Radon Transform of Box Splines): The Radon transform of an $s$-variate box spline specified by the direction set $\boldsymbol{\Xi}$ along a direction $\boldsymbol{\theta}$ is a univariate box spline (i.e., a B-spline along $\boldsymbol{\theta}$ ). The directions (i.e., knots) of the latter univariate box

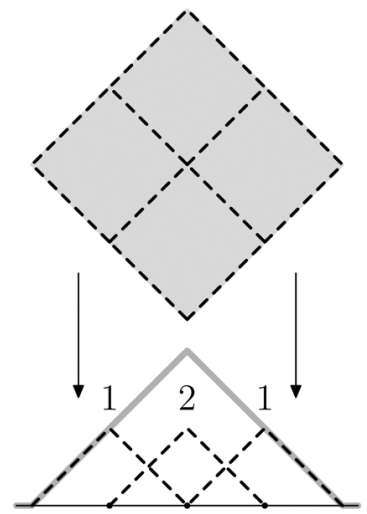

Fig. 3. Natural multiscale relationship for box splines by projection.

spline are obtained by geometric projection of the directions $\xi \in \boldsymbol{\Xi}$ onto the projection direction $\boldsymbol{\theta}$

$$
\zeta=\langle\xi, \theta\rangle \theta
$$

Since any box spline is (geometrically) constructed by the shadow (X-ray) transform of a hypercube, these results establish that the space of box splines are closed under X-ray transform. These results suggest that box splines are suitable basis functions for problems involving tomographic reconstruction.

Another feature of box splines that is particularly useful in the context of tomography is their multiscale property. Since box splines are obtained by projecting a hypercube down to a lower dimensional space, the subdivision of the hypercube leads to a natural formula for a box spline that is written as a sum of scaled versions of itself where the scaled versions are projections of subdivided hypercubes-see Fig. 3.

$$
M_{[11]}(x)=M_{[11]}(2 x-1)+2 M_{[11]}(2 x)+M_{[11]}(2 x+1) .
$$

Since this is based on the subdivision of the $N$-hypercube, this multiscale relationship exists in any dimension $d$ and can be used to develop nonseparable wavelets that use box splines as the scaling functions [16], [9].

\section{EXPLicIt Formulae IN Two DimENSIONS}

For $d=2$, we will now show that the X-ray transforms of box splines are polynomial splines of degree $N-1$. The geometric configuration is the one shown in Fig. 1 with the $x$-to- $y$ projection matrix given by $\mathbf{P}_{\boldsymbol{\theta}^{\perp}}=\left[\begin{array}{ll}\cos \theta & \sin \theta\end{array}\right]$. The application of Theorem 1 together with the convolution formula (10) yields

$$
\mathcal{P}_{\boldsymbol{\theta}} M_{\Xi}(y)=\left(M_{\zeta_{1}} * M_{\zeta_{2}} * \cdots * M_{\zeta_{N}}\right)(y)
$$

with $\zeta_{n}=\mathbf{P}_{\boldsymbol{\theta}^{\perp}} \boldsymbol{\xi}_{n}=\left[\boldsymbol{\xi}_{n}\right]_{1} \cos \theta+\left[\boldsymbol{\xi}_{n}\right]_{2} \sin \theta$, and

$$
M_{\zeta_{n}}(y)=\frac{1}{\zeta_{n}} \operatorname{box}\left(\frac{y}{\zeta_{n}}\right)
$$

which is a rectangular box of width $\zeta_{n}$ when $\zeta_{n} \neq 0$. Note that the convolution factors with $\zeta_{n}=0$ may be eliminated from 
(17) since $M_{0}(y)=\delta(y)$. To evaluate the convolution product, we write $M_{\zeta_{n}}(y)$ as

$$
M_{\zeta_{n}}(y)=\Delta_{\zeta_{n}} u(y)
$$

where $\Delta_{h} f(y)=(f(y)-f(y-h)) /(h)$ is the finite-difference operator with step $h$, and where $u(y)$ is the unit-step (or Heaviside) function. By substituting (18) in (17), we find that

$$
\begin{aligned}
\mathcal{P}_{\boldsymbol{\theta}} M_{\Xi}(y) & =\left(\Delta_{\zeta_{1}} u * \cdots * \Delta_{\zeta_{N}} u\right)(y) \\
& =\frac{\Delta_{\zeta_{1}} \cdots \Delta_{\zeta_{N}} y_{+}^{N-1}}{(N-1) !}
\end{aligned}
$$

where we have used the fact that the $(n-1)$-fold convolution of a step function is $\left(y_{+}^{n}\right) /(n !)$ with $y_{+}^{n}=\max (y, 0)^{n}$. Finally, we may expand the finite-difference operators which yield a linear expansion of $\mathcal{P}_{\boldsymbol{\theta}} M_{\boldsymbol{\Xi}}(y)$ in terms of some shifted versions of $y_{+}^{N-1}$. The result therefore implies that $\mathcal{P}_{\boldsymbol{\theta}} M_{\Xi}(y)$ is a nonuniform polynomial spline of degree $N-1$, or less if some $\zeta_{n}=0$. We can also infer that this box spline function is bell-shaped and that its support is $\sum_{n=1}^{N} \zeta_{n}$.

A case of special interest is when the 2-D basis function (or generator) is a tensor-product B-spline of degree $n: \varphi(\boldsymbol{x})=$ $\beta^{n}\left(x_{1}\right) \beta^{n}\left(x_{2}\right)$ [24]. In the present formalism, this corresponds to a box spline with direction vectors $\boldsymbol{\xi}_{1}=(1,0)$ and $\boldsymbol{\xi}_{2}=$ $(0,1)$, each having a multiplicity $(n+1)$ so that $N=2 n+2$. The specialization of (19) for these particular values yields an explicit formula for the Radon transform of a separable B-spline of degree $n$

$$
\mathcal{P}_{\theta}\left\{\beta^{n}\left(x_{1}\right) \beta^{n}\left(x_{2}\right)\right\}(y)=\frac{\Delta_{\cos \theta}^{n+1} \Delta_{\sin \theta}^{n+1} y_{+}^{2 n+1}}{(2 n+1) !}
$$

which corresponds to the spline bikernel identified by Horbelt et al. in [12]. A Matlab routine for computing the centered versions of these functions is provided in Appendix B.

The more general result (19), which is valid for any 2-D box spline, is new to the best of our knowledge. For instance, the Zwart-Powell element [22] is represented by the box spline directions

$$
\boldsymbol{\Xi}=\left[\begin{array}{cccc}
1 & 0 & 1 & -1 \\
0 & 1 & 1 & 1
\end{array}\right]
$$

According to Theorem 1, its projection along the angle $\theta$ provides the univariate box spline that is specified by the directions

$$
\boldsymbol{Z}=\left[\begin{array}{llll}
\cos \theta & \sin \theta & \cos \theta+\sin \theta & \cos \theta-\sin \theta
\end{array}\right] .
$$

This integration process is illustrated in Fig. 4. By applying (19), we find that the Radon transform of the Zwart-Powell box spline has the explicit closed-form representation

$$
\mathcal{P}_{\theta}\left\{M_{\Xi}\right\}(y)=\frac{\Delta_{\cos \theta} \Delta_{\sin \theta} \Delta_{\cos \theta+\sin \theta} \Delta_{\cos \theta-\sin \theta} y_{+}^{3}}{3 !} .
$$

\section{EXPERIMENTAL RESULTS}

The main interest of the proposed B-spline formalism is to provide an effective and consistent way to discretize the forward

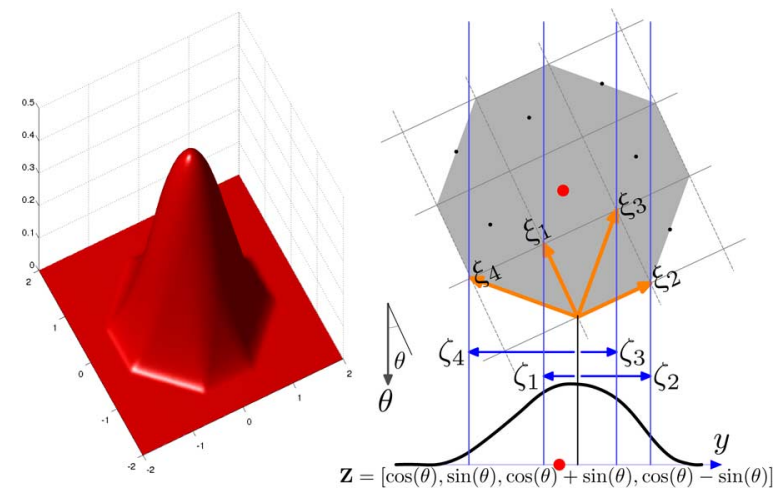

Fig. 4. (Nonseparable) Zwart-Powell element which is a box spline associated with the directions in (21). Radon transform of Zwart-Powell box spline can be derived, exactly, using our approach.

model of a computed-tomography reconstruction problem. The basis for such an approach is to characterize the image (5) by its B-spline coefficients $\mathbf{c}=\left(c_{\boldsymbol{k}}\right)_{\boldsymbol{k} \in \Omega}$ where $\Omega$ denotes the domain of the image and to apply (6) to obtain the simulated line-integral measurements (Radon transform). The image reconstruction is then formulated as a regularized least square optimization problem that is solved iteratively. Specifically, the reconstructed image is given by (5) with the $\mathrm{B}$-spline coefficients being determined as

$$
\mathbf{c}^{\star}=\arg \min _{\mathbf{c}}\|\mathbf{p}-\mathbf{H} \mathbf{c}\|^{2}+\lambda R(\mathbf{c})
$$

where $\mathbf{p}$ is the input measurement vector and $\mathbf{H}$ the matrix-representation of the forward model. The quantity $R(\mathbf{c})$ is a regularization functional (e.g., the energy of the gradient of the image or its total variation) that penalizes nondesirable solutions; it is a way of introducing prior information on the solution to make the problem well-posed. The scalar parameter $\lambda \geq 0$ is a tradeoff factor that balances the fitting accuracy versus the amount of regularization.

The success of such a reconstruction algorithm is dependent upon two factors: 1) the quality and accuracy of the forward model, and 2) the constraints that are imposed by the regularization. The latter is very much application-dependent and becomes especially relevant when the reconstruction problem is ill-posed (e.g., limited angle tomography). Since the appropriate choice of the regularization is a whole field of investigation in itself, we focus here on the assessment of the quality of the forward model.

To that end, we consider a well-conditioned scenario where the measurement noise is negligible and the number of projection angles is sufficient to reconstruct the image by numerical inversion of the forward model. Our series of experiments is set up such that the number of degrees of freedom of the B-spline image model (square grid of size $M \times M$ ) matches the number of measurements ( $M$ properly sampled projections in an equiangular configuration). The reconstruction is performed by solving (23) with $\lambda=0$ iteratively (least squares solution) using the conjugate Gradient (CG) method. We study the accuracy of the family of separable B-spline models as well as the nonseparable Zwart-Powell box spline and compare our method with the traditional implementation of the Radon transform. Because 


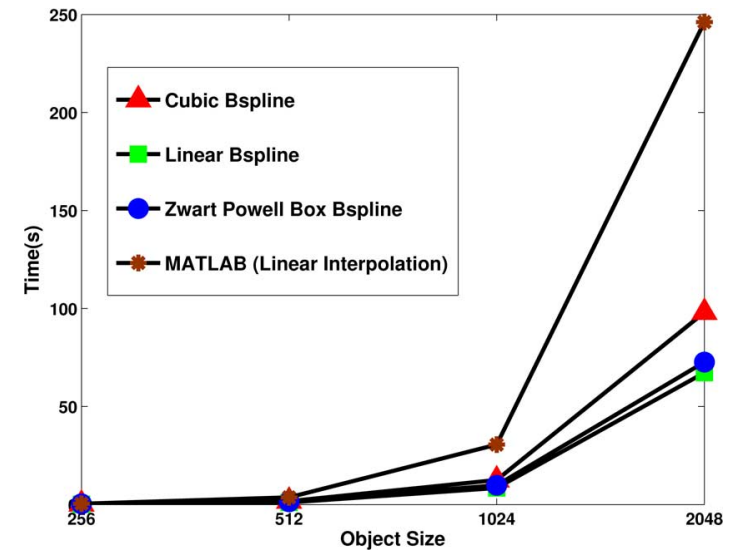

Fig. 5. Comparison of different methods with respect to processing time.

of space limitations, we concentrate on the piecewise linear and cubic B-spline solutions with $n=1,3$ in (20), respectively.

Our reference algorithm is the function radon in Matlab, which proceeds in a hierarchical fashion. It first subdivides each pixel into four subpixels of equal intensity and then projects each of the subcomponents using a triangle profile function $\operatorname{tri}(y)$ (splatting). This implies that the Matlab function is at least four times more demanding than the first-order version (linear B-spline) of our method.

\section{A. Algorithm Speed}

The present family of forward models (B-spline Radon transform) was coded in $\mathrm{C}$ and linked to Matlab as a mex file. The adjoint operator is implemented similar to the forward model by changing the direction of the flow graph. The Radon transform of the B-splines was precomputed once and stored in a 2-D lookup table for best efficiency. To measure the speed, we computed the Radon transforms of a series of images of increasing size $M$. The expected computational cost is $O\left(M^{3}\right)$ with a proportionality factor that depends upon the choice of algorithm and the size of the underlying basis function. The results are documented in Fig. 5 and are consistent with the prediction. The Matlab implementation is the slowest, while the cubic B-spline version of our algorithm is approximately eight times more costly than the piecewise-linear version. The Zwart-Powell box spline is notably faster than cubic B-spline and is very close to linear B-spline's performance.

\section{B. Consistency of Forward Model}

In order to examine the ability of the proposed discretization method to capture sharp image details, we considered an analytical phantom whose Radon transform is known analytically.

Proposition 4: The Radon transform of the function

$$
f(\boldsymbol{x})= \begin{cases}\|\boldsymbol{x}\|^{2}, & \|\boldsymbol{x}\|<a \\ 0, & \text { otherwise }\end{cases}
$$

where $a \in \mathbb{R}^{+}$, is

$$
\mathcal{R}_{\theta}\{f\}(y)= \begin{cases}\frac{2}{3} \sqrt{a^{2}-y^{2}}\left(a^{2}+2 y^{2}\right), & |y|<a \\ 0, & \text { otherwise }\end{cases}
$$
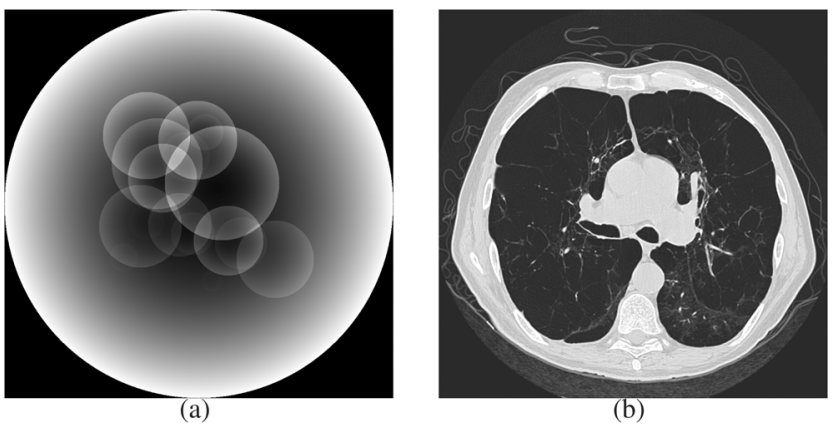

Fig. 6. (a) Analytical phantom includes 30 circles with different quadratic intensity distributions. (b) Lung image used for evaluation of forward model and reconstruction. These datasets at the resolution of $1024 \times 1024$ serve as ground truth for our experiments.

This can be readily verified by evaluating the integral

$$
\mathcal{R}_{\theta}\{f\}(y)=2 \times \int_{0}^{\sqrt{a^{2}-y^{2}}}\left(x^{2}+y^{2}\right) \mathrm{d} x .
$$

Using the linearity and the projected shift-invariance property (4) of the Radon transform, we use this result to determine the Radon transform of an object

$$
I(\boldsymbol{x})=\sum_{\boldsymbol{k}} \alpha_{\boldsymbol{k}} f\left(\boldsymbol{x}-\boldsymbol{x}_{\boldsymbol{k}}\right)
$$

where $\alpha_{\boldsymbol{k}} \in \mathbb{R}$ and $\boldsymbol{x}_{\boldsymbol{k}} \in \mathbb{R}^{2}$ are some prescribed parameters. For our experiments, we considered the analytical phantom shown in Fig. 6(a). Starting from a $1024 \times 1024$ representation, we calculated its Radon transform along 1024 directions with the help of the different algorithms and compared the output with the analytical one. Examples of projections are shown in Fig. 7. The higher order versions of our spline models produce the sinograms that are the most faithful to the analytical ones. The Matlab results in 7(c) are not quite as favorable as the cubic B-spline 7(e) and Zwart-Powell box spline 7(d), although they oscillate less than the linear spline version 7(b). This ranking is confirmed by the global signal-to-noise ratio (SNR) presented in Table I.

\section{Reconstruction Error as a Function of Grid Size}

In a well-conditioned scenario with a sufficient number of measurements, we can expect the quality of the reconstruction to be dependent upon the grid size (number of degrees of freedom of the reconstruction model). Yet, we also know from approximation theory that not all basis functions are equally good at representing arbitrary signals at a fixed resolution. From a sampling point of view, B-splines are optimal in the sense that they have the maximal order of approximation for a given support.

To investigate the dependence upon the sampling rate, we conducted a series of experiments where the grid size is progressively reduced. The reference object and signal-to-noise computations are defined with respect to the fine grid (e.g., $1024 \times 1024)$. The coarse grid measurements are obtained by suitable angular and spatial resampling of the fine-grid Radon transform of the object. An ideal lowpass filter is applied in the spatial domain prior to downsampling to avoid aliasing. The object is reconstructed on the coarse grid using the different 


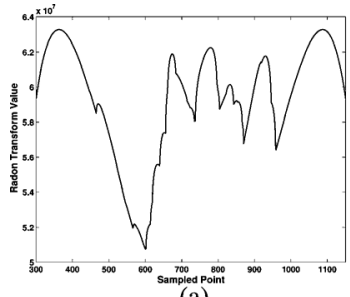

(a)

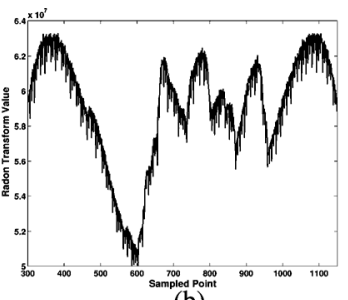

(b)

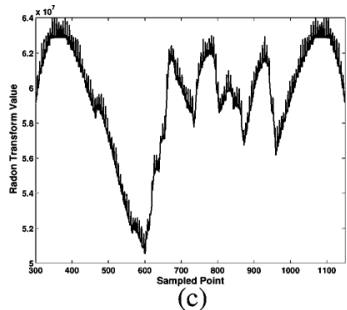

(c)
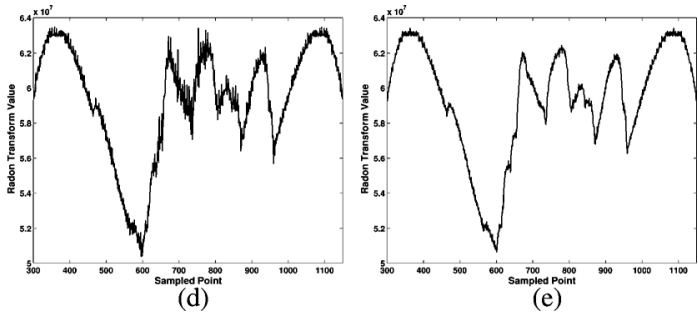

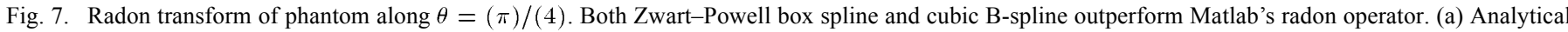
Radon transform, (b) linear B-spline, (c) Matlab radon operator, (d) Zwart-Powell box spline, and (e) cubic B-spline.

TABLE I

COMPARISOn OF DifFERENT Discrete MODEls of RADON TRANSForm

\begin{tabular}{c||c|c|c|c} 
Method & MATLAB & Degree1 & Zwart-Powell & Degree3 \\
\hline SNR(dB) & 43.85 & 39.88 & 44.65 & 52.75
\end{tabular}

flavors of the forward model. The reconstruction is calculated iteratively and corresponds to the least-squares solution. Finally, the result is interpolated back to the finer grid (resampling of the spline model) for quality assessment. For the Matlab version, we used a linear interpolation which was found to give better results than a cubic interpolation (for upsampling).

1) Analytical Phantom for Analysis of Accuracy: In the case of the phantom in Fig. 6(a), we used the analytical calculation of the Radon transform as initial fine-grid measurements. We then performed the various signal reconstructions for $M=1024,512,256$, and 128 using the corresponding downsampled versions of the input data. The evolution of the signal-to-noise ratio as a function of the downsampling factor is shown in Fig. 8. Fig. 9 compares the reconstruction results for a central region of the phantom that is reconstructed from 256 projections (downsampling by $(4,4)$ ). The specific region of the phantom is depicted in Fig. 9(a). Note that the best results are obtained with the cubic B-spline [Fig. 9(e)] and Zwart-Powell box spline [Fig. 9(d)] models. In particular, we can distinguish some of the fainter circles (e.g., at the bottom left) that are barely visible in the other reconstructions. The differences between the Matlab model and the linear B-spline reconstruction are less significant, although there may be a slight preference towards the former because of its smoother appearance.

2) Biomedical Data: Next, we considered the cross section of the lung image shown in Fig. 6(b) as representative example of a medical image. Its Radon transform was calculated using the different forward models and the results averaged to specify a fine-scale set of measurements that is not biased towards one of the methods. We then performed the same experiments as in the previous case. The corresponding evolution of the SNR is shown in Fig. 10. Fig. 11 presents a region of interest that was reconstructed from 256 projections. The conclusions that can be drawn are essentially the same as in the previous experiment, namely, that the cubic B-spline [Fig. 11(e)] and Zwart-Powell box spline [Fig. 11(d)] basis functions outperforms the others. This is significantly and of practical interest, given the computational efficiency of these two algorithms (see Fig. 5).

In addition, we did also reconstruct images using the quintic $\mathrm{B}$-spline version of the method which is computationally more

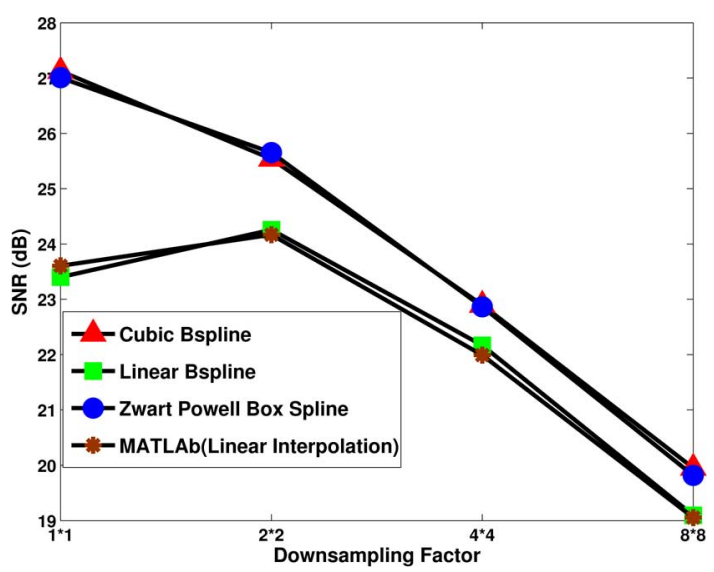

Fig. 8. Evolution of the signal-to-noise ratio for least squares reconstruction of analytical phantom as both grid size and number of measurements are reduced.

expensive, but did not observe any significant improvement over the cubic spline reconstruction which appears to offer an excellent compromise in terms of cost/quality tradeoff. We believe that the present cubic B-spline and Zwart-Powell box spline versions of the Radon transform are to be preferred over the standard Matlab implementation because they consistently yield better quality results while being computationally quite competitive.

We also performed experiments with real biomedical data (i.e., human bicuspid calcific heart valve derived nodule) acquired from a CT scanner. The computational advantages of our spline model as well as improvements in the accuracy of reconstruction, afforded by higher order basis functions, were similar to the lung data experiments reported in Fig. 11. Moreover, we have performed an experiment with a phantom containing sharp edges to assess the impact of higher-order basis functions on the sharpness of reconstruction. This experiment illustrates, numerically, that the higher-order basis functions do provide a more accurate reconstruction with a better preservation of edges. The results and discussions of these experiments are documented in the supplementary material for this manuscript. ${ }^{2}$

\section{DISCUSSION}

The choice of the box spline generator in (5) should be determined by computational and approximation theoretic considerations. Basis functions with larger support and smoothness usually offer better approximation quality, but they also

\footnotetext{
${ }^{2}$ Available at http://www.cise.ufl.edu/ entezari/research/radon-transform/ supp.pdf.
} 


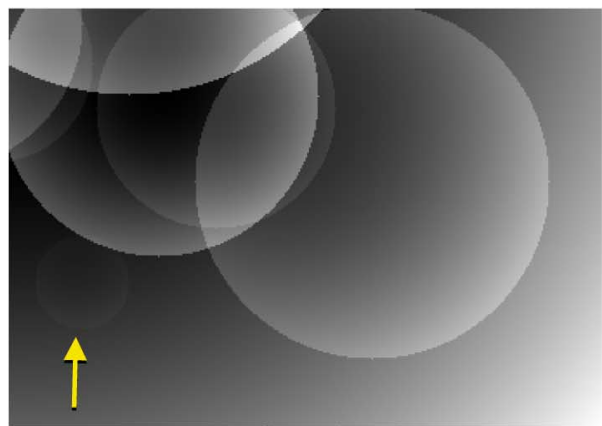

(a) Ground Truth

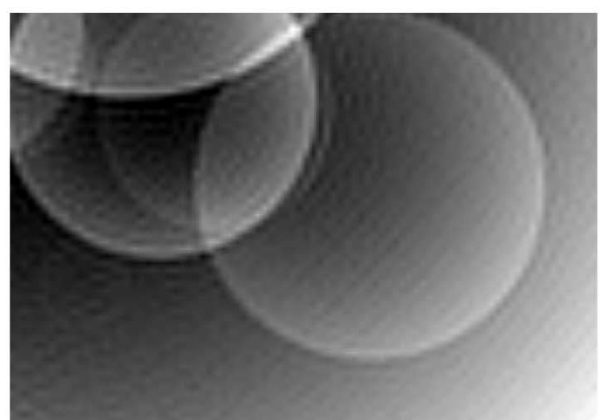

(b) Matlab model SNR=27.06 dB

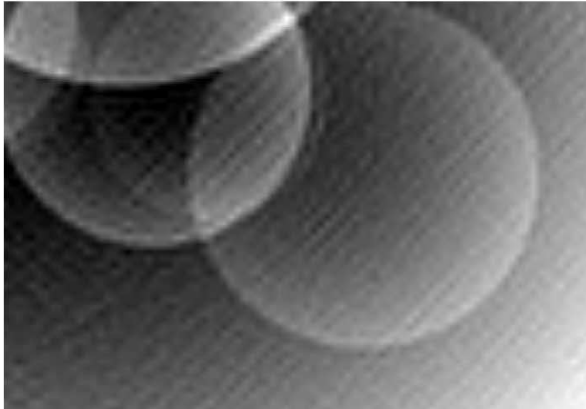

(c) Linear B-spline SNR=25.83 dB

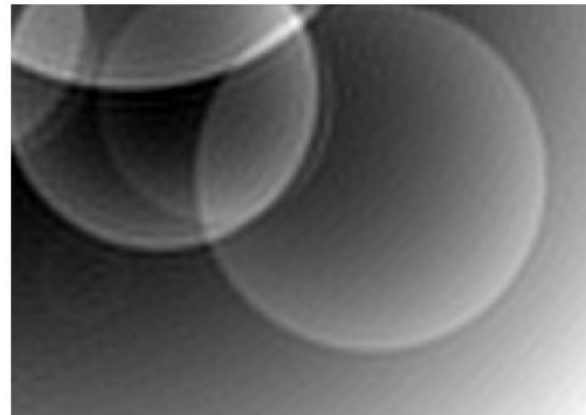

(d) Zwart-Powell box spline $\mathrm{SNR}=28.04 \mathrm{~dB}$

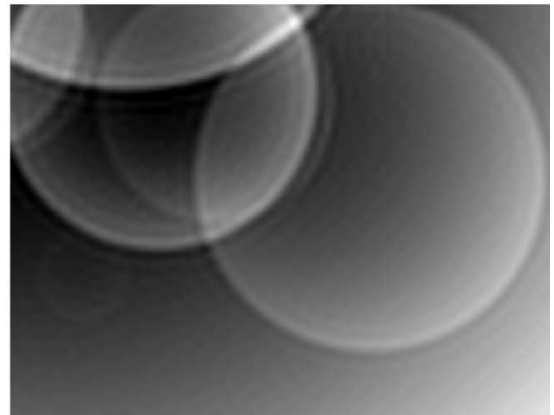

(e) Cubic B-spline SNR=28.22 dB

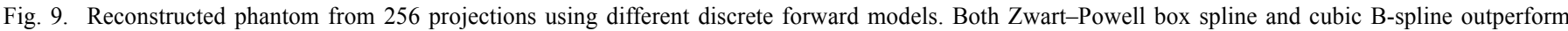
Matlab's reconstruction. Besides having smaller artifacts in the reconstruction, the faint circle in the bottom left is better reconstructed in (d) and (e).

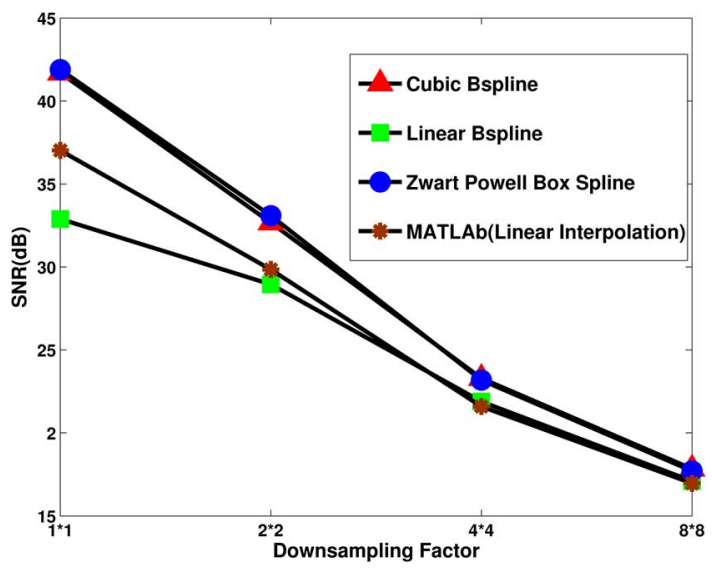

Fig. 10. Evolution of the signal-to-noise ratio for least squares reconstruction of the lung as both grid size and number of measurements are reduced.

require more computations. This suggests the possibility of a tradeoff between approximation order and the density of the reconstruction grid. In particular, it has been demonstrated that it is computationally advantageous asymptotically to switch to a higher order basis function than to increase the sampling rate [12].

Tensor-product B-splines constitute a preferred set of basis functions because they are made up from univariate B-splines building blocks which are widely studied and efficient to evaluate. We should note, however, that the present box spline framework includes other nonseparable basis functions with increased isotropy (e.g., Zwart-Powell box spline) and same approximation order, but lower polynomial degree and smaller support than their tensor-product counterparts. This directly relates to the efficiency issue (Fig. 5), which deserves further investigation.

The approach is applicable with minor adjustment to nonparallel geometries as long as the projections are line integrals or obtained by taking "point" measurements (i.e., ideal samples) of the Radon transform, or, with a very slight extension, that all the rays hitting one detector (pixel) are parallel. Since the Radon transform of the B-spline/box spline is available to us in closed form, the handling of a nonparallel geometry then essentially amounts to a proper bookkeeping of the angles. For every ray angle in the nonparallel geometry, the proper ray direction in the parallel geometry can be looked up. The hypothesis of pure line integrals is implicit to all the discretization methods that we are aware of.

Another concept for consideration is the geometry of the reconstruction lattice. While the common solution is to use a Cartesian grid, the 2-D hexagonal lattice and the 3-D body centered cubic (BCC) and face centered cubic (FCC) lattices have been demonstrated to outperform the sampling efficiency of the Cartesian lattice due to their densest spectral packing properties. Advantages of hexagonal, BCC, and FCC lattices for tomography have been advocated for discretizing image space [11], [8], [15], [21], [10]. The present box spline formalism can perfectly handle these lattices as well as the separable Cartesian lattice. Equation (5) can model lattice shifts of a box spline that is geometrically tailored for such nonseparable lattices. The BCC/FCC box splines investigated in [5], [3], and [13] can be readily applied since these are quite favorable from the computational aspects. Moreover, the Voronoi splines [18] and hex-splines [25] are composed of box splines; hence, the 


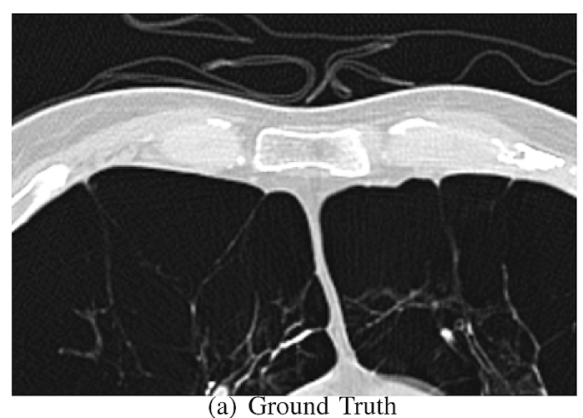

(a) Ground Truth

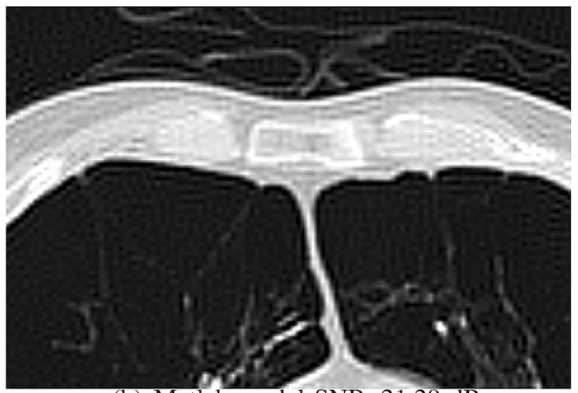

(b) Matlab model SNR=21.29 dB

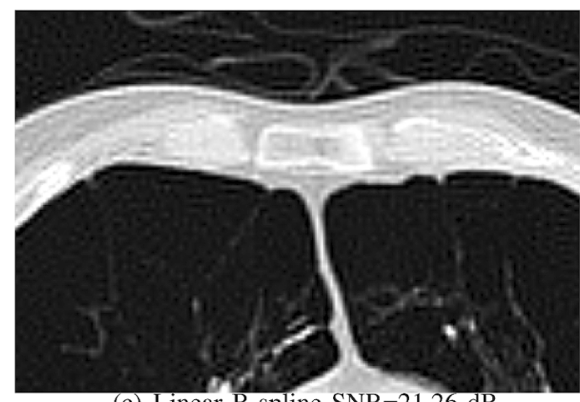

(c) Linear B-spline SNR=21.26 dB

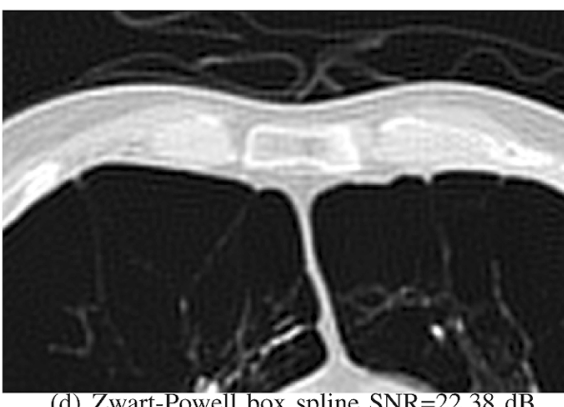

(d) Zwart-Powell box spline SNR=22.38 dB

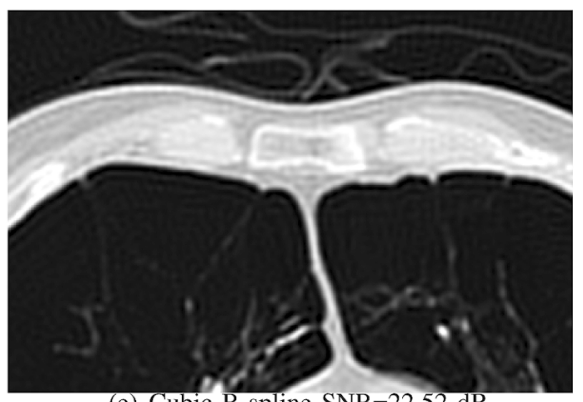

(e) Cubic B-spline SNR=22.52 dB

Fig. 11. Central region of reconstructed lung from 256 projections using different discrete forward models. Linear B-spline (c) is comparable to Matlab's approach (b), while both Zwart-Powell box spline (d) and Cubic B-spline (e) provide the most accurate reconstructions. Difference in the reconstructions are mostly visible in areas close to edges as well as the white structures within gray areas.

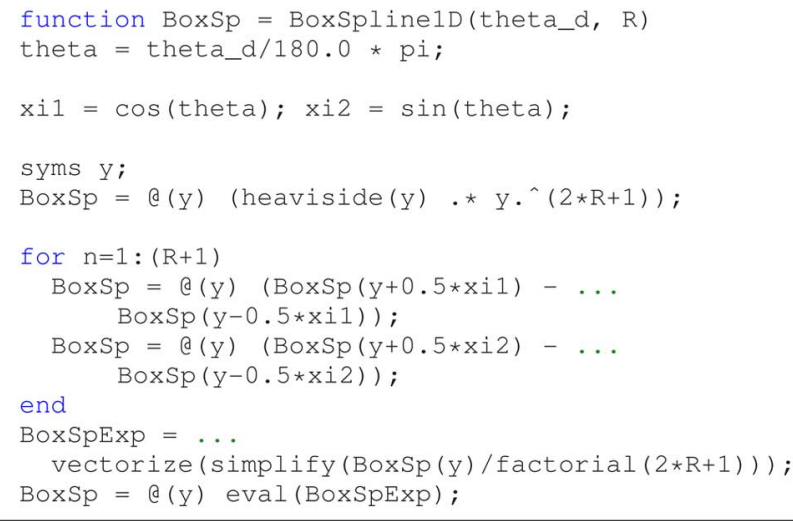

Fig. 12. Matlab code for Radon transform of the tensor-product B-splines.

framework discussed in this paper can be extended to the class of Voronoi splines for reconstruction on arbitrary lattices.

\section{CONCLUSION}

We presented a box spline calculus for computed tomography. The proposed framework translates the X-ray and Radon transforms to geometric projections applied on the direction sets of box splines. While the framework is general for separable and nonseparable box splines in any dimension, the 2-D cases are studied specifically for the Zwart-Powell box spline and the commonly used tensor-product B-splines. The X-ray transforms of tensor-product B-splines of any order lead to nonseparable box splines whose closed-form representations are provided along with efficient Matlab routines for their implementation.

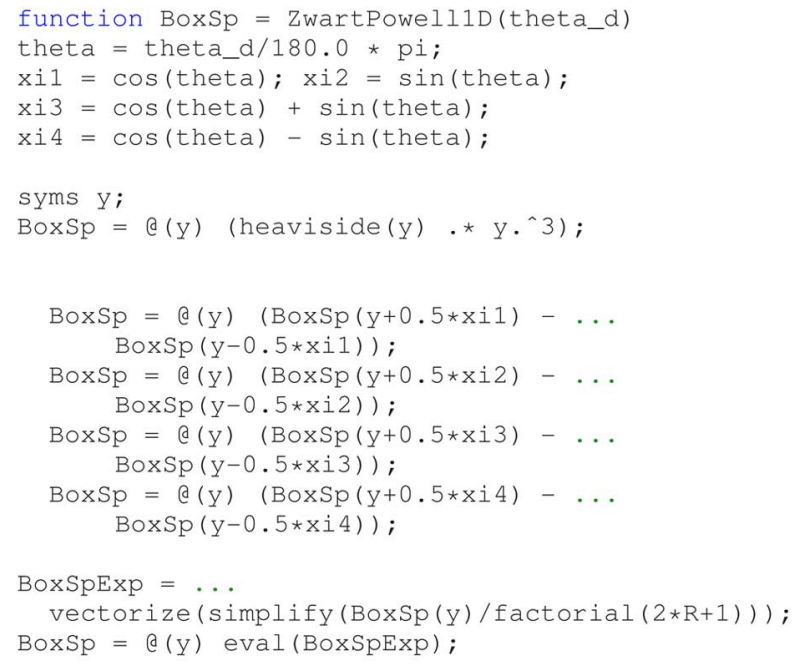

Fig. 13. Matlab code for Radon transform of the Zwart-Powell box spline.

\section{APPENDIX A \\ MATLAB IMPLEMENTATION}

The 2-D to 1-D X-ray transform of a B-spline can be computed by (20) which can be efficiently implemented in Matlab, as shown in Fig. 12.

Similarly, the 2-D to 1-D X-ray transform of the nonseparable Zwart-Powell box spline can be computed by (22) which can be efficiently implemented in Matlab, as shown in Fig. 13.

\section{REFERENCES}

[1] B. Bojanov and Y. Xu, "Reconstruction of a polynomial from its radon projections," SIAM J. Math. Anal., vol. 37, no. 1, pp. 238-250, 2005. 
[2] C. de Boor, K. Höllig, and S. Riemenschneider, Box Splines, Volume 98 of Applied Mathematical Sciences. New York: Springer-Verlag, 1993.

[3] A. Entezari, R. Dyer, and T. Möller, "Linear and cubic box splines for the body centered cubic lattice," in Proc. IEEE Conf. Visualizat., Oct. 2004, pp. 11-18.

[4] A. Entezari and M. Unser, "A box spline calculus for computed tomography," in Proc. 2010 IEEE Int. Symp. Biomed. Imag.: From Nano to Macro, Apr. 2010, pp. 600-603.

[5] A. Entezari, D. Van De Ville, and T. Möller, "Practical box splines for reconstruction on the body centered cubic lattice," IEEE Trans. Visualizat. Comp. Graph., vol. 14, no. 2, pp. 313-328, Mar.-Apr. 2008.

[6] A. S. Fokas, A. Iserles, and V. Marinakis, "Reconstruction algorithm for single photon emission computed tomography and its numerical implementation," J. R. Soc. Interface, vol. 3, no. 6, pp. 45-54, 2006.

[7] A. S. Fokas and V. Marinakis, "Reconstruction algorithm for the brain imaging techniques of PET and SPECT," in Adv. Develop. 1994-2005, 2006, p. 443.

[8] E. Garduno and G. T. Herman, "Optimization of basis functions for both reconstruction and visualization," Discrete Appl. Math., vol. 139, no. 1-3, pp. 95-111, 2004.

[9] W. He and M. Lai, "Construction of trivariate compactly supported biorthogonal box spline wavelets," J. Approx. Theory, vol. 120, no. 1, pp. 1-19, 2003.

[10] G. T. Herman, Geometry of Digital Spaces. Boston, MA: Birkhauser, 1998.

[11] G. T. Herman, Fundamentals of Computerized Tomography: Image Reconstruction From Projections. New York: Springer, 2009.

[12] S. Horbelt, M. Liebling, and M. Unser, "Discretization of the radon transform and of its inverse by spline convolutions," IEEE Trans. Med. Imag., vol. 21, no. 4, pp. 363-376, Apr. 2002.

[13] M. Kim, A. Entezari, and J. Peters, "Box spline reconstruction on the face centered cubic lattice," IEEE Trans. Visualizat. Comp. Graph., vol. 14 , no. 6 , pp. $1523-1530$, Jun. 2008
[14] M. Lautsch, "A spline inversion formula for the radon transform," SIAM J. Numer. Anal., vol. 26, no. 2, pp. 456-467, 1989.

[15] S. Matej and R. Lewitt, "Practical considerations for 3-D image reconstruction using spherically symmetric volume elements," IEEE Trans. Med. Imag., vol. 15, no. 1, pp. 68-78, Jan. 1996.

[16] S. Meignen, "Application of the convergence of the control net of box splines to scale-space filtering," IEEE Trans. Image Process., vol. 16, no. 11 , pp. $2842-2848$, Nov. 2007.

[17] E. Meijering, "Quantitative evaluation of convolution-based methods for medical image interpolation," Med. Img. Anal., vol. 5, no. 2, pp. 111-126, Jun. 2001.

[18] M. Mirzargar and A. Entezari, "Voronoi splines," IEEE Trans. Signal Process., vol. 58, no. 9, pp. 4572-4582, Sep. 2010.

[19] F. Natterer, "The mathematics of computerized tomography," in Proc. SIAM, Philadelphia, PA, 2001

[20] X. Pan, E. Y. Sidky, and M. Vannier, "Why do commercial CT scanners still employ traditional, filtered back-projection for image reconstruction?," Inverse Problems, vol. 25, no. 12, p. 123009, 2009.

[21] P. Rattey and A. Lindgren, "Sampling the 2-D Radon transform," IEEE Trans. Acoustics, Speech, Signal Process., vol. 29, no. 5, pp. 994-1002, May 1981.

[22] M. Richter, "Use of box splines in computer tomography," Computing, vol. 61 , no. 2 , pp. $133-150,1998$

[23] P. Thévenaz, T. Blu, and M. Unser, "Interpolation revisited," IEEE Trans. Med. Imag., vol. 19, no. 7, pp. 739-758, Jul. 2000.

[24] M. Unser, A. Aldroubi, and M. Eden, "B-spline signal processing: Part I-Theory," IEEE Trans. Signal Process., vol. 41, no. 2, pp. 821-833, Feb. 1993.

[25] D. Van De Ville, T. Blu, M. Unser, W. Philips, I. Lemahieu, and R. Van de Walle, "Hex-splines: A novel spline family for hexagonal lattices," IEEE Trans. Image Process., vol. 13, no. 6, pp. 758-772, Jun. 2004.

[26] Y.Xu, "Reconstruction from Radon projections and orthogonal expansion on a ball," J. Physics A: Math. Theoretical, vol. 40, p. 7239, 2007. 